\title{
Co-infection of SARS-COV-2 and Influenza A Virus: A Case Series and Fast Review*
}

\author{
Xuan XIANG, Zi-hao WANG, Lin-lin YE, Xin-liang HE, Xiao-shan WEI, Yan-ling MA, Hui LI, Long CHEN, \\ Xiao-rong WANG, Qiong ZHOU ${ }^{\#}$ \\ Department of Respiratory and Critical Care Medicine, Union Hospital, Tongji Medical College, Huazhong University of \\ Science and Technology, Wuhan 430022, China
}

(C) Huazhong University of Science and Technology 2021

\begin{abstract}
Summary: Coronavirus disease 2019 (COVID-19) occurs in the influenza season and has become a global pandemic. The present study aimed to examine severe acute respiratory syndrome coronavirus 2 (SARS-CoV-2) co-infection with influenza A virus (IAV) in an attempt to provide clues for the antiviral interventions of co-infected patients. We described two patients who were co-infected with SARS-CoV-2 and IAV treated at Wuhan Union Hospital, China. In addition, we performed a review in PubMed, Web of Science and CNKI (from January 1 up to November 1, 2020) with combinations of the following key words: "COVID-19, SARS-COV-2, influenza A and co-infection". A total of 28 co-infected patients were enrolled in the analysis. Of the 28 patients, the median age was 54.5 years (IQR, 34.25-67.5) and 14 cases $(50.0 \%)$ were classified as severe types. The most common symptoms were fever $(85.71 \%)$, cough $(82.14 \%)$ and dyspnea $(60.71 \%)$. Sixteen patients had lymphocytopenia on admission and 23 patients exhibited abnormal radiological changes. The median time from symptom onset to hospital admission was 4 days (IQR, 3-6), and the median time of hospital stay was 14 days (IQR, 8.5-16.75). In conclusion, patients with SARSCOV-2 and IAV co-infection were similar to those infected with SARS-COV-2 alone in symptoms and radiological images. SARS-COV-2 co-infection with IAV could lead to more severe clinical condition but did not experience longer hospital stay compared with patients infected with SARSCOV-2 alone.
\end{abstract}

Key words: co-infection; COVID-19; influenza A; SARS-COV-2

A new acute respiratory disease occurred in the winter of 2019, and was named coronavirus disease 2019 (COVID-19). It was found to be caused by severe acute respiratory syndrome coronavirus 2 (SARSCoV-2). SARS-CoV-2 falls within the subgenus Sarbecovirus of the genus Betacoronavirus, with about $79 \%$ identity to SARS-associated coronavirus (SARS-CoV) and sharing a similar receptor-binding domain structure to that of SARS-CoV ${ }^{[1]}$. SARSCoV-2 now has swept the world again with a stunning speed and brought tremendous pressure to the medical system. The clinical characteristics and radiological changes of COVID-19 have been well documented. The most common symptoms of COVID-19 are fever and cough ${ }^{[2,3]}$, and the most representative images of computed tomography are peripheral ground-glass

Xuan XIANG, E-mail: xxuan@hust.edu.cn

\#Corresponding author, E-mail: zhouqiongtj@126.com

${ }^{*}$ This work was supported by the National Natural Science Foundation of China (No. 81973990, No. 81900096, and No.81770090) and Fundamental Research Funds for the Central Universities (No. 2020kfyXGYJ030). opacities $^{[4,5]}$.

Influenza A viruses (IAV) belong to the Orthomyxoviridae family and are further divided into subtypes according to the two surface glycoproteins, haemagglutinin and neuraminidase. The acute respiratory illnesses caused by IAV usually occur in winter and lead to economic loss around the world ${ }^{[6,7]}$. Based on the features of host and virus, patients infected with IAV could present different symptoms, including fever, chills, myalgia and respiratory symptoms such as dry cough, nasal congestion, etc. The typical changes in chest radiology images of IAV patients are groundglass opacities and consolidation $\mathbf{S}^{[8,9]}$.

Viral-bacterial and viral-viral co-infection are common during the influenza season and may lead to severe clinical illness ${ }^{[10,11]}$. There have been a number of patients infected with both SARS-CoV-2 and IAV in the COVID-19 pandemic ${ }^{[12-14]}$. Due to the sporadic cases, the characteristics and course of those coinfected patients remain unknown. In the present study, we reviewed all published studies and described a cohort of patients co-infected with SARS-CoV-2 and IAV in the COVID-19 pandemic, aiming to improve 
the management of these co-infection patients.

\section{MATERIALS AND METHODS}

\subsection{Search Strategy and Study Selection}

We retrospectively screened out two patients infected with SARS-CoV-2 and IAV in 145 confirmed COVID-19 patients in Wuhan Union Hospital, China, and oral consents were obtained from the two patients. Then we searched PubMed (National Library of
Medicine, Washington, DC), Web of Science and China National Knowledge Infrastructure (CNKI) from January 1 up to November 1, 2020, using different combinations of the following key words: "COVID-19, SARS-CoV-2, influenza A and co-infection". We also reviewed the reference lists of retrieved articles to search for other proper studies. All retrievals were publications concerning human studies in English or Chinese. The flow chart of searching and selecting the studies is shown in fig. 1.

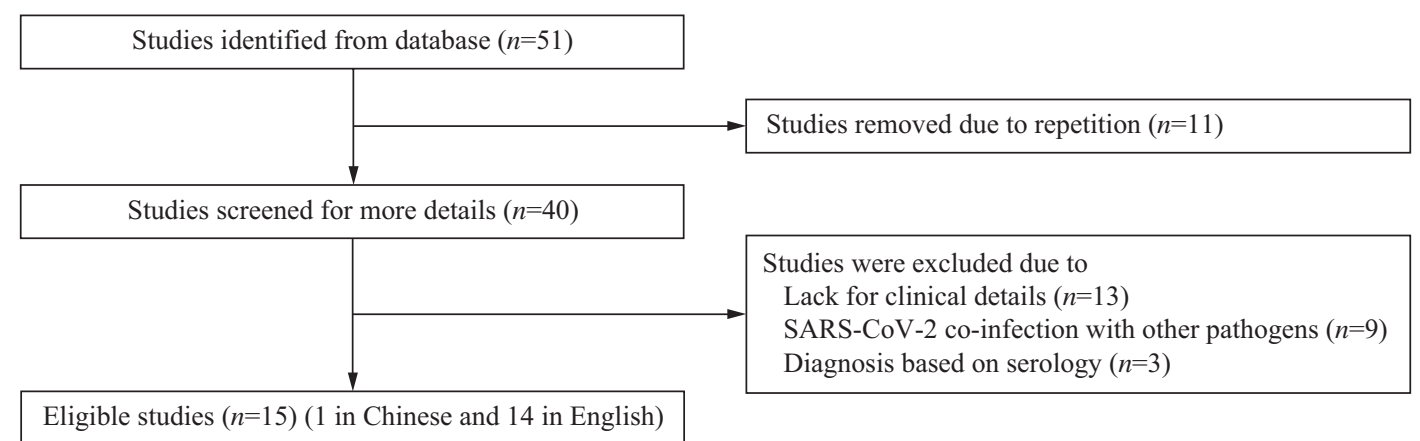

Fig. 1 The process of searching and selecting the studies

Inclusive criteria were as follows: (1) studies on SARS-CoV-2 and IAV co-infection; (2) case report on SARS-CoV-2 and IAV co-infection. Exclusion criteria were: (1) studies not containing any information on clinical course of co-infected patients; (2) studies on SARS-CoV-2 co-infection with other pathogens; (3) SARS-CoV-2 or IAV infection diagnosed based on serum antibody.

\subsection{Data Extraction}

For each study, the following information was extracted: the author's last name; year; country; study design; number of patents reported; number of patents co-infected with SARS-CoV-2 and IAV; selected clinical data [age; gender; comorbidities; symptoms; days from symptom onset to hospital admission; days in hospital; lymphocyte count on admission; oxygen saturation on admission; radiological images after admission; treatment; clinical events, including acute respiratory distress syndrome (ARDS), data about noninvasive ventilation, endotracheal intubation, and intensive care unit (ICU); clinical outcome (improved or death)]. Patients were divided into moderate type (showing fever and respiratory symptoms with radiological findings of pneumonia) and severe type (severe type was defined if one of the following conditions was met: 1 . respiratory distress, respiratory rate $\geq 30$ per $\min ; 2$. oxygen saturation on room air at rest $\leq 93 \% ; 3$. partial pressure of oxygen in arterial blood/fraction of inspired oxygen $\leq 300 \mathrm{mmHg}$ ) according to the Novel Coronavirus Pneumonia Diagnosis and Treatment Plan (trial version 7) developed by the National Health Committee of the People's Republic of China ${ }^{[15]}$.

\subsection{Statistical Analysis}

Frequency with percentages (\%) was used to describe categorical variables. Continuous variables were described using median and interquartile range (IQR) values. All statistical analyses were performed with GraphPad Prism 8.0.2.

\section{RESULTS}

\subsection{Study Screening}

After independent review, 40 publications on SARS-CoV-2 and IAV co-infection were considered eligible for inclusion in the analysis. Of these publications, 13 were excluded for not having detailed clinical information on patients co-infected with SARSCOV-2 and IAV, and 9 for SARS-CoV-2 co-infection with other pathogens. We also excluded 3 studies in which IAV diagnosis was based on serology. It is not reliable to diagnose IAV infection by only serological results ${ }^{[16]}$. The remaining 15 publications (one searched from CNKI and 14 from PubMed) provided 26 cases with clinical information. Therefor 28 patients who suffered from both SARS-CoV-2 and IAV co-infection were enrolled in the analysis ${ }^{[13,14,17-29]}$ (table 1).

\subsection{Clinical Characteristics of Patients with SARS -CoV-2 and IAV Co-infection}

Table 2 summarizes the demographics and clinical characteristics of the total 28 patients. In this descriptive study, 14 patients $(50.0 \%)$ were classified as severe cases and $16(57.1 \%)$ were males. The median age was 54.5 years (IQR, 34.25-67.5). Thirteen patients had comorbidities such as hypertension, 
Table 1 Study samples included in the review

\begin{tabular}{|c|c|c|c|c|}
\hline Authors, year, country & Type of study & Case number & $\begin{array}{l}\text { No. of SARS-COV-2 } \\
\text { co-infection with IAV }\end{array}$ & $\begin{array}{c}\text { Diagnostic method } \\
\text { of IAV }\end{array}$ \\
\hline Data from our hospital, 2020, China ${ }^{*}$ & Original data & 145 & 2 & NAA \\
\hline Wu et al, 2020, China ${ }^{[13]}$ & Case report & 1 & 1 & NAA \\
\hline Khodamoradi et al, 2020, $\operatorname{Iran}^{[14]}$ & Case report & 4 & 4 & NAA \\
\hline Azekawa et al, 2020, Japan ${ }^{[25]}$ & Case report & 1 & 1 & NAA \\
\hline Konala et al, 2020, USA ${ }^{[24]}$ & Case report & 1 & 1 & NAA \\
\hline Konala et al, 2020, $\mathrm{USA}^{[18]}$ & Case report & 3 & 3 & NAA \\
\hline Sha, 2020, China ${ }^{[17]}$ & Case report & 1 & 1 & NAA \\
\hline Cuadrado-Payán et al, 2020, Spain ${ }^{[28]}$ & Case report & 4 & 2 & NAA \\
\hline Wehl et al, 2020, Germany ${ }^{[21]}$ & Short communication & 1 & 1 & RICAT \\
\hline Ozaras et al, 2020, Turkey ${ }^{[27]}$ & Research article & 1103 & 2 & DFA \\
\hline Singh et al, 2020, USA ${ }^{[26]}$ & Case report & 3 & 1 & RAA \\
\hline Kakuya et al, 2020, Japan ${ }^{[29]}$ & Short communication & 3 & 1 & $\mathrm{CI}$ \\
\hline Zheng et al, 2020, China ${ }^{[22]}$ & Letter to the Editor & 1001 & 4 & NAA \\
\hline D'Abramo et al, 2020, Italy ${ }^{[19]}$ & Case report & 1 & 1 & NAA \\
\hline Pongpirul et al, 2020, Thailand ${ }^{[20]}$ & Clinical study & 11 & 1 & NAA \\
\hline Hashemi et al, 2020, $\operatorname{Iran}^{[23]}$ & Letter to the Editor & 600 & 2 & NAA \\
\hline
\end{tabular}

NAA: nucleic acid amplification; DFA: direct fluorescent antigen; RAA: rapid antigen assay; CI: chromatographic immunoassay; RICAT: rapid immune chromatographic assay testing. * Data were collected from Wuhan Union Hospital.

diabetes, tumor, cerebrovascular disease, coronary artery disease, dyslipidemia, hypothyroidism, pituitary microadenoma, congestive heart failure, chronic kidney disease and chronic lung disease. The most common symptoms before admission were fever $(85.7 \%)$, cough (82.1\%), dyspnea (60.7\%) and myalgia (46.15\%).

On admission, $69.6 \%$ of the patients had decreased lymphocyte count and 23 patients $(85.2 \%)$ had abnormal radiological changes in their first examination after admission. The median time from symptom onset to hospital admission was 4 days (IQR, 3-6), and the median time of hospital stay was 14 days (IQR, 8.5-16.75). 50.0\% patients had lower oxygen saturation $\left(\mathrm{SpO}_{2} \leq 93 \%\right)$ on ambient air. $54.6 \%$ of these patients had oxygen inhalation, including five patients receiving noninvasive ventilation, and five endotracheal intubation in the ICU. $64.3 \%$ had oseltamivir treatment, and $73.9 \%$ had other antiviral treatment except oseltamivir. $79.3 \%$ received antibiotic therapy, and only $8.7 \%$ were treated with corticosteroids. Six patients suffered from ARDS and three patients failed surviving the pandemic. The clinical condition of 18 patients was improved after admission. The outcomes of the remaining seven patients, however, were not available.

\section{DISCUSSION}

Viruses co-infection usually occurs in the influenza epidemic and pandemic ${ }^{[30,31]}$. Previous studies found that co-infection with IAV and other viruses significantly increased the risk of admission to a general ward, and IAV and influenza B virus coinfection increased the risk of admission to ICU or death $^{[30]}$. During the COVID-19 pandemic, numerous studies have reported co-infection of SARS-CoV-2 and IAV. To better understand the clinical course of patients co-infected with SARS-CoV-2 and IAV, we performed a fast review on these patients.

In this review, we collected 28 patients co-infected with SARS-CoV-2 and IAV from China $\left.{ }^{[13}, 17,22\right]$, $\operatorname{Japan}^{[25,29]} \operatorname{Iran}^{[14,23]}$, America ${ }^{[18,24,26]}$, Germany ${ }^{[21]}$ and

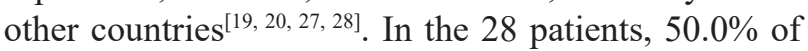
patients were severe type and $14.3 \%$ died, which were higher than those reported in COVID-19 ${ }^{[32]}$. Moreover, a report from Iran showed $22.3 \%$ of dead cases were coinfected with SARS-CoV-2 and IAV ${ }^{[33]}$, indicating coinfection with SARS-CoV-2 and IAV may result in more severe condition. IAV infection was more common in children and old people ${ }^{[6]}$. We only found three children suffering from SARS-CoV-2 and IAV co-infection in our cohort ${ }^{[17,21,29]}$. The clinical manifestations of patients co-infected with SARS-CoV-2 and IAV are similar to those infected with SARS-CoV-2 alone, such as fever, cough, dyspnea and myalgia, etc. However, in this review, we found $60.7 \%$ patients had dyspnea on admission, higher than that reported by Wang and Huang et al $(31.2 \% \text { and } 55 \% \text { respectively })^{[2,3]}$. The median time of hospital stay in this review was 14 days (IQR, 8.5-16.75), shorter than the previous finding (median, 18 days, IQR, 14-27) ${ }^{[34]}$. These data indicate that patients co-infected with SARS-CoV-2 and IAV developed more severe clinical condition, while having shorter hospital stay. We speculate that one important reason may be attributed to the timely use of effective antiviral drugs such as oseltamivi. $64.3 \%$ of patients were treated with oseltamivi in this cohort, which could limit the propagation of IAV infection and reduce 


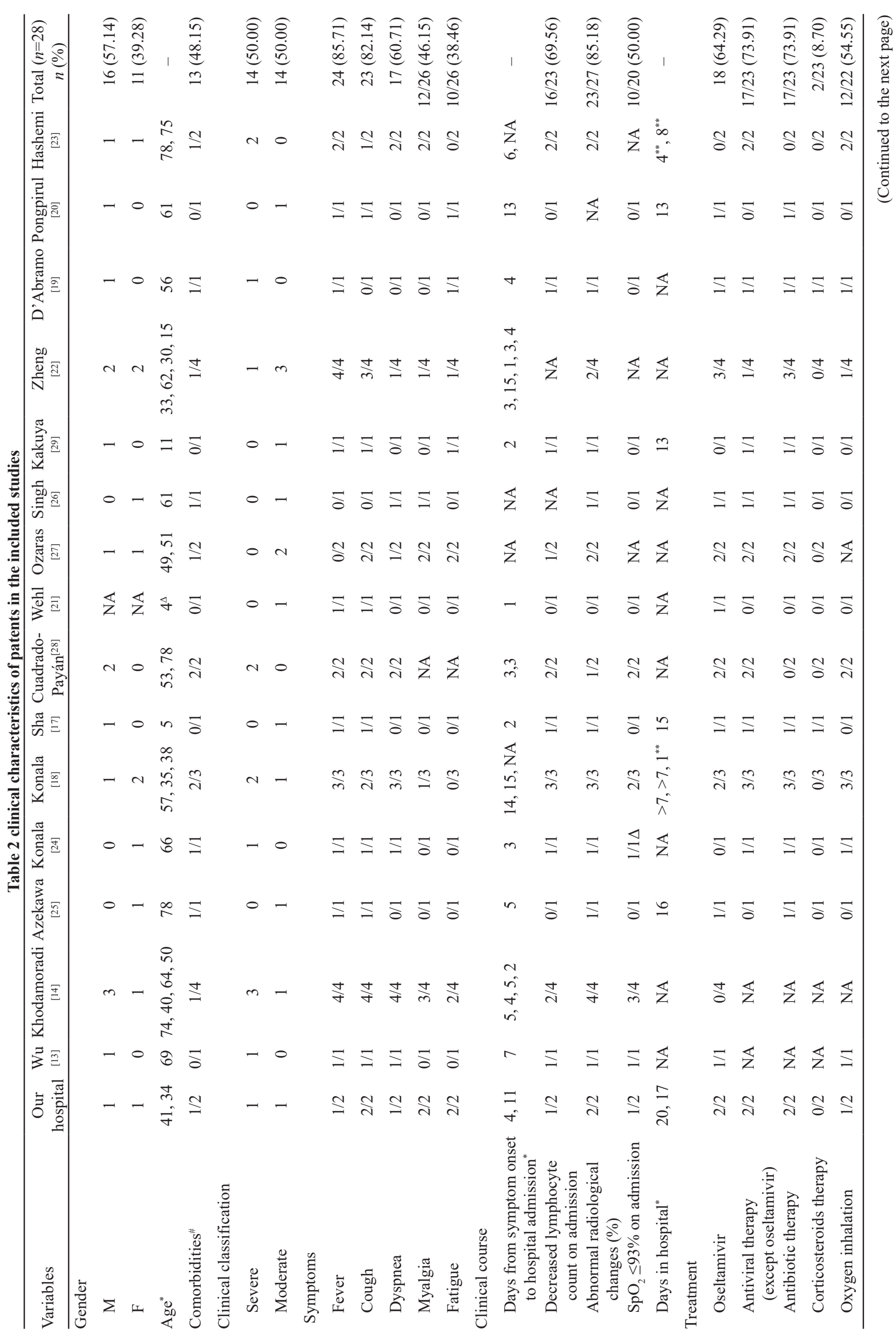


the risk of complications, especially when dealing with lower respiratory tract complications ${ }^{[35]}$. On the other hand, some interesting interference may occur in two viruses co-infection ${ }^{[36]}$. $85.2 \%$ of patients exhibited typical abnormal radiological changes and $69.56 \%$ of patients showed decreased lymphocyte count on admission, which could indicate viral infection but could not distinguish exact pathogens.

In this review, we found 18 patients $(64.3 \%)$ were treated with oseltamivir, a neuraminidase inhibitor recommended to deal with influenza by the European Union and the USA ${ }^{[37,38]}$, and $73.9 \%$ patients received antibiotic therapy to prevent secondary bacterial infection ${ }^{[39]}$. However, whether oseltamivir has effect on SARS-CoV-2 viral shedding and whether coinfection with SARS-CoV-2 and IAV may cause secondary infection need further investigation. Two patients were treated with corticosteroids, while corticosteroids were not usually recommended in the COVID-19 pandemic concerning about their possible side effects on virus clearance and their association with high rates of complications ${ }^{[40,41]}$. Eighteen patients were improved and, unfortunately, three patients lost their life in this study.

We noticed that patients co-infected with SARSCoV-2 and IAV accounted for a small proportion in the COVID-19 pandemic ${ }^{[42,43}$, which may be due to the interference between viruses ${ }^{[4]}$. The mechanism of SARS-CoV-2 and IAV interference remains to be further clarified.

Admittedly, this study has some limitations. First and most, the sample size is extremely small due to scanty reports on SARS-CoV-2 and IAV co-infection. Second, we could not collect detailed information about each patient since some information was not available, and, additionally, the missing information may have some influence on our results. Third, the reports did not provide SARS-CoV-2 viral shedding time, and thus whether co-infection with IAV has some influence on SARS-CoV-2 viral shedding remains unknown. Fourth, we could not identify which virus was first infected, which may help account for the co-infection course. Finally, lower respiratory tract samples were not harvested for detection of SARS-CoV-2 and IAV in these studies included, and therefore whether the two viruses were both involved in the pathological progression of pulmonary remains unclear.

In conclusion, our study found that patients with SARS-CoV-2 and IAV co-infection were similar to those infected with SARS-CoV-2 alone in symptoms and radiological images. Although patients with SARS$\mathrm{CoV}-2$ and IAV co-infection did not experience longer hospital stay than those infected with SARS-CoV-2 alone, they usually presented with more severe clinical condition. Physicians should pay more attention to the patients co-infected with two or more respiratory 
pathogens.

\section{Conflict of Interest Statement}

The authors declare that there is no conflict of interest with any financial organization or corporation or individual that can inappropriately influence this work.

\section{REFERENCES}

1 Lu R, Zhao X, Li J, et al. Genomic characterisation and epidemiology of 2019 novel coronavirus: implications for virus origins and receptor binding. Lancet, 2020,395(10224):565-574

2 Wang $\mathrm{D}, \mathrm{Hu} \mathrm{B}, \mathrm{Hu} \mathrm{C}$, et al. Clinical Characteristics of 138 Hospitalized Patients With 2019 Novel Coronavirus-Infected Pneumonia in Wuhan, China. JAMA, 2020,323(11):1061

3 Huang C, Wang Y, Li X, et al. Clinical features of patients infected with 2019 novel coronavirus in Wuhan, China. Lancet, 2020,395(10223):497-506

4 Chung M, Bernheim A, Mei X, et al. CT Imaging Features of 2019 Novel Coronavirus (2019-nCoV). Radiology, 2020:200230

5 Zhang R, Ouyang H, Fu L, et al. CT features of SARS$\mathrm{CoV}-2$ pneumonia according to clinical presentation: a retrospective analysis of 120 consecutive patients from Wuhan city. Eur Radiol, 2020,30(8):4417-4426

6 Thompson WW. Influenza-Associated Hospitalizations in the United States. JAMA, 2004,292(11):1333

7 Lafond KE, Nair H, Rasooly MH, et al. Global Role and Burden of Influenza in Pediatric Respiratory Hospitalizations, 1982-2012: A Systematic Analysis. Plos Med, 2016,13(3):e1001977

8 Collins JP, Campbell AP, Openo K, et al. Clinical Features and Outcomes of Immunocompromised Children Hospitalized with Laboratory-Confirmed Influenza in the United States, 2011-2015. J Pediat Inf Dis Soc, 2019,8(6):539-549

9 Ercen DO, Arslan S, Akdogan O, et al. Clinical, radiological and prognostic features of influenza cases in the influenza epidemic during years 2016-2017. Tuberk Toraks, 2018,66(2):144-199

10 McCullers JA. Insights into the Interaction between Influenza Virus and Pneumococcus. Clin Microbiol Rev, 2006,19(3):571-582

11 Martin-Loeches I, J Schultz M, Vincent J, et al. Increased incidence of co-infection in critically ill patients with influenza. Intens Care Med, 2017,43(1):48-58

12 Ding Q, Lu P, Fan Y, et al. The clinical characteristics of pneumonia patients coinfected with 2019 novel coronavirus and influenza virus in Wuhan, China. J Med Virol, 2020,92(9):1549-1555

13 Wu X, Cai Y, Huang X, et al. Co-infection with SARS$\mathrm{CoV}-2$ and Influenza A Virus in Patient with Pneumonia, China. Emerg Infect Dis, 2020,26(6):1324-1326

14 Khodamoradi Z, Moghadami M, Lotfi M. Co-infection of Coronavirus Disease 2019 and Influenza A: A Report from Iran. Arch Iran Med, 2020,23(4):239-243

15 National Health Commission, State Administration of Traditional Chinese Medicine. Diagnosis and Treatment of Protocol of COVID-19. http://www.nhc.gov.cn/ xcs/zhengcwj/202003/46c9294a7dfe4cef80dc7f5912 eb1989.Shtml, 2020- 03-03
16 Uyeki TM. Influenza. Ann Intern Med, 2017,167(5): C33-C48

17 Sha GJ. Co-infection of SARS CoV-2 and influenza A in a child: a case report. Henan J Prev Med (Chinese), 2020,31(05): 323-324

18 Konala VM, Adapa S, Naramala S, et al. A Case Series of Patients Coinfected With Influenza and COVID-19. J Investig Med High Impact Case Rep, 2020,8:1562230171

19 D'Abramo A, Lepore L, Palazzolo C, et al. Acute respiratory distress syndrome due to SARS-CoV-2 and Influenza A co-infection in an Italian patient: Minireview of the literature. Int J Infect Dis, 2020,97:236239

20 Pongpirul WA, Mott JA, Woodring JV, et al. Clinical Characteristics of Patients Hospitalized with Coronavirus Disease, Thailand. Emerg Infect Dis, 2020,26(7):15801585

21 Wehl G, Laible M, Rauchenzauner M. Co-infection of SARS CoV-2 and influenza A in a Pediatric Patient in Germany. Klin Padiatr, 2020,232(4):217

22 Zheng X, Wang H, Su Z, et al. Co-infection of SARSCoV-2 and Influenza virus in Early Stage of the COVID-19 Epidemic in Wuhan, China. J Infect, 2020, 81(2):e128-e129

23 Hashemi SA, Safamanesh S, Ghafouri M, et al. Coinfection with COVID-19 andinfluenza A virus in two died patients with acute respiratory syndrome, Bojnurd, Iran. J Med Virol, 2020,92(11):2319-2321

24 Konala VM, Adapa S, Gayam V, et al. Co-infection with Influenza A and COVID-19. European J Case Rep Intern Med, 2020,7(5):1

25 Azekawa S, Namkoong H, Mitamura K, et al. Coinfection with SARS-CoV-2 and influenza A virus. IDCases, 2020,20:e775

26 Singh B, Kaur P, Reid R, et al. COVID-19 and Influenza Co-Infection: Report of Three Cases. Cureus, 2020,12(8): e9852

27 Ozaras R, Cirpin R, Duran A, et al. Influenza and COVID-19 coinfection: Report of six cases and review of the literature. J Med Virol, 2020,92(11):2657-2665

28 Cuadrado-Payán E, Montagud-Marrahi E, Torres-Elorza $\mathrm{M}$, et al. SARS-CoV-2 and influenza virus co-infection. Lancet, 2020,395(10236):e84

29 Kakuya F, Okubo H, Fujiyasu H, et al. The First Pediatric Patients with Coronavirus Disease 2019 (COVID-19) in Japan: Risk of Co-Infection with Other Respiratory Viruses. Jpn J Infect Dis, 2020,73(5):377-380

30 Goka E, Vallely P, Mutton K, et al. Influenza A viruses dual and multiple infections with other respiratory viruses and risk of hospitalisation and mortality. Influenza Other Resp, 2013,7(6):1079-1087

31 Meskill SD, Revell PA, Chandramohan L, et al. Prevalence of co-infection between respiratory syncytial virus and influenza in children. Am J Emerg Med, 2017,35(3): 495-498

32 Guan W, Ni Z, Hu Y, et al. Clinical Characteristics of Coronavirus Disease 2019 in China. New Engl J Med, 2020,382(18):1708-1720

33 Hashemi SA, Safamanesh S, Ghasemzadeh-Moghaddam $\mathrm{H}$, et al. High prevalence of SARS-CoV-2 and influenza A virus (H1N1) coinfection in dead patients in 
Northeastern Iran. J Med Virol, 2020,93:1008-1012

$34 \mathrm{Xu} \mathrm{K}$, Chen Y, Yuan J, et al. Factors associated with prolonged viral RNA shedding in patients with COVID-19. Clin Infect Dis, 2020,71(15):799-806

35 Dobson J, Whitley RJ, Pocock S, et al. Oseltamivir treatment for influenza in adults: a meta-analysis of randomised controlled trials. Lancet, 2015,385(9979): 1729-1737

36 Pinky L, Dobrovolny HM. SARS-CoV-2 coinfections: Could influenza and the common cold be beneficial? J Med Virol, 2020,92(11):2623-2630

37 European Centre For Disease Control. Scientific-adviceneuraminidase-inhibitors-2017. https://www.ecdc.europa. eu/sites/default/files/documents/Scientific-adviceneuraminidase-inhibitors-2017.

38 Centers for Disease Control and Prevention. Influenza, https://www.cdc.gov/flu/treatment/whatyoushould.htm

39 McCullers JA, Bartmess KC. Role of Neuraminidase in Lethal Synergism between Influenza Virus and
Streptococcus pneumoniae. J Infect Dis, 2003,187(6): 1000-1009

40 Arabi YM, Mandourah Y, Al-Hameed F, et al. Corticosteroid Therapy for Critically Ill Patients with Middle East Respiratory Syndrome. Am J Resp Crit Care, 2018,197(6):757-767

41 Stockman LJ, Bellamy R, Garner P. SARS: systematic review of treatment effects. Plos Med, 2006,3(9):e343

42 Nowak MD, Sordillo EM, Gitman MR, et al. Coinfection in SARS-CoV-2 infected Patients: Where Are Influenza Virus and Rhinovirus/Enterovirus? J Med Virol, 2020,92(10):1699-1700

43 Kim D, Quinn J, Pinsky B, et al. Rates of Co-infection between SARS-CoV-2 and Other Respiratory Pathogens. JAMA, 2020,323(20):2085-2086

44 Schultz-Cherry S. Viral Interference: The Case of Influenza Viruses. J Infect Dis, 2015,212(11):1690-1691 (Received Jun. 8, 2020; accepted Jan. 14, 2021) 